\title{
Examining student understanding of matrix algebra and eigentheory
}

Pachi Her and Michael Loverude

Department of Physics, California State University, Fullerton, 800 N State College Blvd, Fullerton, CA 92831

Matrix algebra and eigentheory are important to students' learning of physics in upper division physics courses. In this study, we investigated how students apply matrix algebra and eigentheory in various physics contexts, excluding quantum mechanics. The data collected consist of student written responses taken from a math methods course. We used the Physical-Mathematical Model (Uhden et al., 2011) to categorize each question by the three skills the model presents: mathematization, interpretation, and technical operation. The results from our data show that students have difficulty with mathematizing and interpreting the mathematical and physical system of a matrix equation, but are fluent in technical operations. We will present examples of student responses illustrating student reasoning, and discuss implications for classroom instruction.

2020 PERC Proceedings edited by Wolf, Bennett, and Frank; Peer-reviewed, doi.org/10.1119/perc.2020.pr.Her Published by the American Association of Physics Teachers under a Creative Commons Attribution 4.0 license. Further distribution must maintain the cover page and attribution to the article's authors. 


\section{INTRODUCTION}

Mathematics plays a key role in describing the properties of a physical system. Physics students encounter increasingly diverse and difficult mathematics as they move into upperdivision courses, and the successful comprehension of these concepts is critical to their understanding of physics. This paper will focus on student understanding of matrix algebra and eigentheory from linear algebra; it explores student written responses to identify the extent and nature of student difficulty with these two topics.

\section{A. Previous Research}

Several studies across multiple fields have found that linear algebra is a difficult subject for students. Within the Physics Education Research (PER) community, there has been limited research on student understanding of linear algebra application in physics. Most of these studies focus on the context of quantum mechanics [1]. Sabella and Redish summarized various research papers that generally stated "students usually master the algorithmic skills involved in linear algebra, but lack a conceptual understanding of the subject and how to apply linear algebra concepts to physical systems" [2].

While there is little research within the PER community, the Research in Undergraduate Mathematics Education community, RUME, provides a majority of the available research on student understanding of linear algebra. Work by Wawro et al. has investigated meta-representational competence in quantum mechanics problems that involve linear algebra concepts; this work is an interdisciplinary collaboration to investigate the use of linear algebra in quantum mechanics [3]. A study by Larson and Zandeih described a framework describing three different interpretations of matrix equations: linear combination, system of equations, and transformation [4]. This framework complemented Larson's previous study of student conceptualization and interpretation of matrix multiplication [5]. The Linear Algebra Curriculum Study Group, formed by Carlson et al. is a group that focus on improving their courses to increase student comprehension [6]. Additionally, the application of linear algebra has increasingly demonstrated the importance in the understanding and usage of symbols, resulting in several investigations of semiotics in eigentheory $[7,8]$.

There are theoretical works that do exist in the PER community that describe the use of mathematical tools in physics. Redish and Kuo presented a model that describes the structure of how mathematics is used in physics and other sciences [9]. This model follows a four-step process that transitions between understanding the physical system to its mathematical system. A framework known as ACER introduces a method for organizing and extracting students' conceptual knowledge and use of mathematical tools [10]. For this paper we have chosen to analyze student responses using the Physical-Mathematical Model created by Uhden et al. [11], described further below. This model provides a distinction between structural skills of mathematization and interpretation and the technical skill of mathematical operation, and highlights the relationship between the math and physics involved. Our intent was to search for areas in which students struggle the most when applying matrix algebra and eigentheory in various contexts of physics.

\section{B. Motivation for Study}

As stated previously, only a few studies exist that examine linear algebra in the contexts of physics, most of which are in the context of quantum mechanics. We chose contexts of physics other than quantum mechanics in order to complement and contrast with existing work. Thus, further investigation of this research topic can prove to be valuable to researchers, instructors, and students. In addition, several studies have investigated some student understanding of matrix algebra and eigentheory in non-physics contexts as mentioned previously. Investigation of student understanding of these topics in physics context can also shed light on the interface of math and physics.

\section{RESEARCH METHODS}

This research was conducted at California State University Fullerton (CSUF), a Hispanic-serving institution with nearly 40,000 students. The data collected were written responses from students in an upper-division math methods physics course. Generally, the enrollment for this course ranges to about 7 to 18 students per semester. This sophomore-junior level course teaches students various mathematical concepts to prepare students for future upper division physics courses. The students enrolled have taken at least three semesters of calculus and at least two lower division physics courses. Over several semesters, approximately two-thirds of the students reported prior completion of a linear algebra course.

\section{A. Task Design}

There are three main tasks that will be the primary focus for this paper. These tasks were given on seven exams from four different semesters taught by the same instructor. Prior to taking these exams, the students completed tutorials, homework assignments, and a quiz related to matrix algebra and eigentheory. In addition, two important equations that the students learned before taking the exams were (1) the eigenequation and (2) the characteristic equation.

$$
\begin{gathered}
\hat{A} \mathbf{x}=\lambda \mathbf{x} \\
\operatorname{det}(\hat{A}-\lambda \hat{I})=0
\end{gathered}
$$


Task 1: Task 1 involved a system of two masses connected by springs (see Fig. 1). The question was divided into two parts. For part A, students were asked to translate the system of equations into a matrix equation. They were expected to take the second order derivatives of $x_{1}=A_{1} e^{i \omega t}$ and $x_{2}=$ $A_{2} e^{i \omega t}$, take the real components, and substitute back into the system of equations. The construction of the matrix equation revealed that it was an eigenequation and students could thus solve for the eigenvalues in order to determine the frequencies as requested in part $\mathrm{B}$.

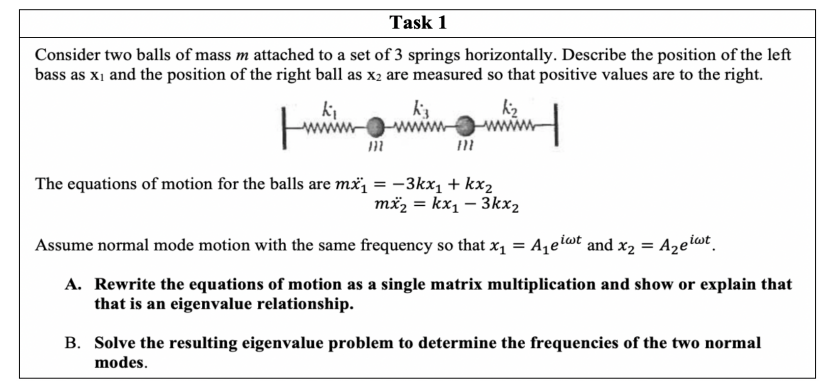

FIG. 1. Task 1 given in four mid-term exams.

A potential issue with Task 1 is the dependence of part B on part A. If students do not construct the matrix equation properly in part A, they will have a difficult time performing the appropriate operations to find the eigenvalues if there is no matrix equation to begin with. This dependency of parts made it difficult to analyze the responses effectively. Thus, the two parts were divided into two separate questions, Tasks 2 and 3, which were given on the final exams for three of the semesters.

Task 2: Task 2 is parallel to part B of Task 1, where it asked for the frequencies of the normal mode springs system (see Fig 2). This time the question gave a matrix equation, similar to the one that the students were expected to construct in Task 1 part A.

Task 2

In a normal mode problem with two springs, the following eigenvalue equation arises:

$$
\left(\begin{array}{cc}
-3 k & k \\
k & -3 k
\end{array}\right)\left(\begin{array}{l}
x_{1} \\
x_{2}
\end{array}\right)=m \omega^{2}\left(\begin{array}{l}
x_{1} \\
x_{2}
\end{array}\right)
$$

Determine the frequencies of the two modes.

FIG. 2. Task 2 given on final exams for three of the semesters.

Task 3: Task 3 is parallel to Task $1 \mathrm{~A}$, asking for a matrix equation, but also asking students to describe how the constructed matrix equation fits the profile of an eigenequation (see Fig 3). In addition, the system was changed from a springs system to a coupled LC circuit.

\section{B. Classification}

Before examining the written responses, we used the Physical-Mathematical Model by Uhden et al. to categorize each task as one or more of the following skills: mathematization, interpretation, or technical operation (see Fig

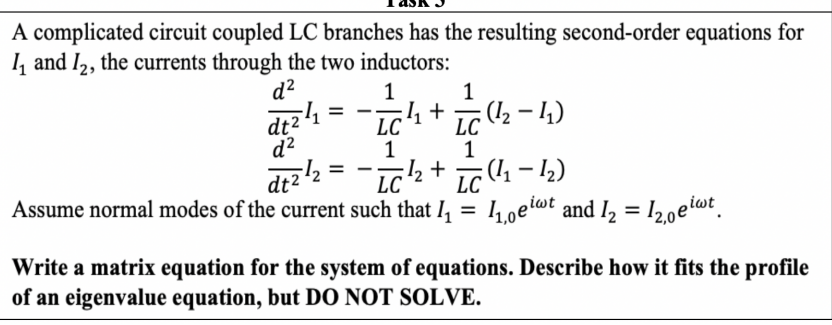

FIG. 3. Task 3 given on final exams for three of the semesters

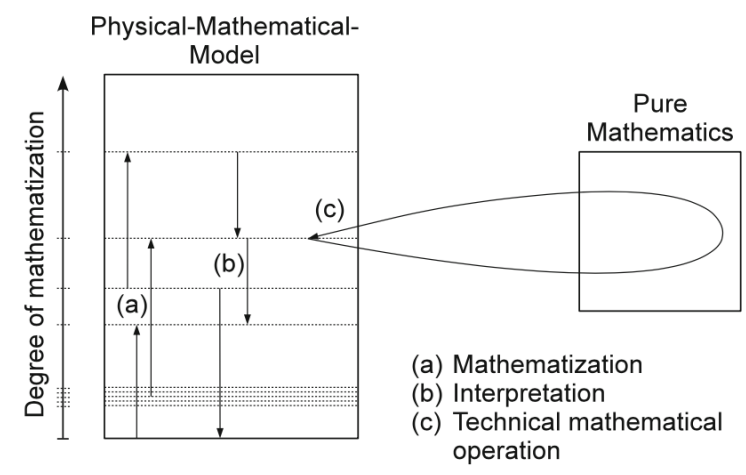

FIG. 4. Representation of the Physical-Mathematical Model created by Uhden et al. It presents the three skills: mathematization, interpretation, and technical operation. Mathematization and interpretation are classified as the structural skills, technical operation as the technical skill.

4). Mathematization and interpretation are characterized as the structural skills, technical operation as the technical skill. Each skill is distinguished based on the degree of mathematization, i.e., the level of mathematical abstraction. The degree of mathematization can increase or decrease depending on the content of the task. For example, in Tasks 1A and 3, students translate a system of equations into a matrix equation. The task presents coupled linear differential equations, so the starting point is already fairly abstract, near the middle of the Physical-Mathematical Model. We claim that the process of translating to the matrix equation increases the degree of mathematization. Figure 5 presents our categorization of each task into its respective skill(s) and degree of mathematization.

We identified that Tasks 1B and 2 incorporated both of the interpretation and technical operation skills. The interpretation skill is the ability to read equations and properly identify the physical meaning and importance of symbols involved in a physical system. Technical operation is described as pure mathematical calculations-the ability to operate and properly use algorithmic rules to perform any required calculations. Note, this skill does not involve any of the structural skills like the other two when performing the calculations; hence, the model indicates a separate mathematical space as seen in Figure 4. For both tasks, the students had to apply technical operation to solve for the eigenvalues, then plug it into $\lambda$ and isolate the frequency. Interpretation was when the students 
TABLE I. Description of each skill's general characteristics along with an example of a student written response

\begin{tabular}{|c|c|c|}
\hline Skills & Characteristics & Example \\
\hline Mathematization & $\begin{array}{l}\text { Translation into } \\
\text { mathematical terms } \\
\text { (e.g., an equation) } \\
\text { Mathematical sense } \\
\text { making - provide an } \\
\text { analogy and reasoning } \\
\text { between mathematical } \\
\text { and physical systems }\end{array}$ & $\begin{aligned} &-m w^{2} x_{1}=-4 k x_{1}+k x_{2} \\
& m w^{2} x_{1}=3 k x_{1}-5 k x_{2} \\
&-m w^{2}\left(\begin{array}{l}x_{1} \\
x_{2}\end{array}\right)=x_{1}\left(\begin{array}{c}-4 k \\
3 k\end{array}\right)+x_{2}\left(\begin{array}{c}k \\
-3 k\end{array}\right)=\left(\begin{array}{cc}-4 k & k \\
3 k & -3 k\end{array}\right)\left(\begin{array}{l}x_{1} \\
x_{2}\end{array}\right) \\
&-m w^{2}\left(\begin{array}{l}x_{1} \\
x_{2}\end{array}\right)=-k\left(\begin{array}{cc}4 & -1 \\
-3 & 3\end{array}\right)\left(\begin{array}{l}x_{1} \\
x_{2}\end{array}\right)\end{aligned}$ \\
\hline Interpretation & $\begin{array}{l}\text { - } \text { "Reading" Equations } \\
\text { Evaluating the } \\
\text { meaning of the } \\
\text { expression, symbols, } \\
\text { and values }\end{array}$ & $\begin{array}{l}\lambda_{1}=\frac{7+\sqrt{13}}{2} \\
\frac{m \omega_{1}^{2}}{k}=\frac{7+\sqrt{13}}{2}\end{array}$ \\
\hline $\begin{array}{l}\text { Technical } \\
\text { Operation }\end{array}$ & $\begin{array}{l}\text { Calculating for } \\
\text { unknown values } \\
\text { Use of operations } \\
\text { (multiplication, } \\
\text { division, subtraction, } \\
\text { and addition) and } \\
\text { equations }\end{array}$ & $\operatorname{det}\left|\begin{array}{cc}4-\lambda & -1 \\
-3 & 3-\lambda\end{array}\right|=(4-\lambda)(3-\lambda)-3=12-7 \lambda+\lambda^{2}-3=\lambda^{2}-7 \lambda+9=0$ \\
\hline
\end{tabular}

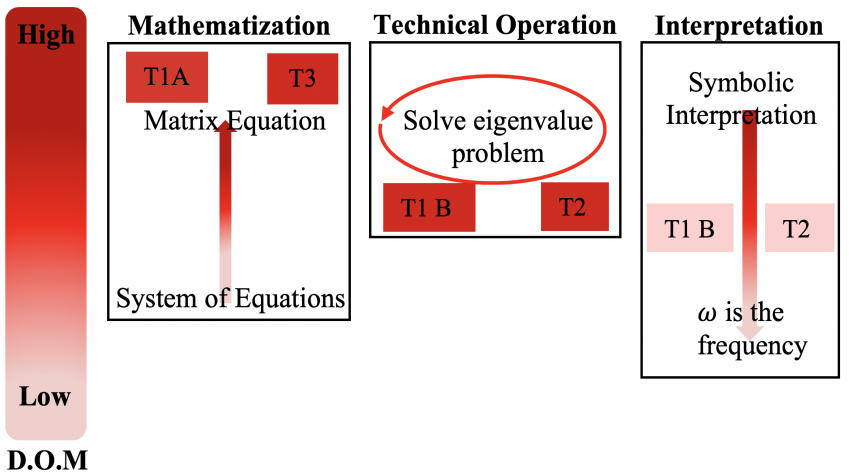

FIG. 5. Categorization of each task into their respective skills and indication of their Degree of Mathematization (D.O.M)

had to identify what $\lambda$ was equal to, and define the symbol that represented the frequency. Table I provides a more indepth description of what each skill represents along with a student example.

\section{Analysis Procedures}

For Task 1A, we coded all 53 responses and looked for answers that followed the structure of a general matrix equation: $\hat{A} \mathbf{x}=\mathbf{b}$. Then, we re-evaluated the responses looking for the "correctness" of the equation. This corresponds to following the structure of the eigenequation along with having the appropriate values for each element presented in the equation. We used the same investigation method for Task 3.
Tasks $1 \mathrm{~B}$ and 2 were analyzed differently because they both involved the calculation for the eigenvalues and the interpretation of the frequencies. We focused on three areas when analyzing the students' responses to these two tasks:

1. Execution of the necessary mathematical operations.

2. Calculation of the correct eigenvalues.

3. Symbolically interpreting the frequency correctly.

It is arguable that factor 2 is fused into factor 1 as both constitute the execution of mathematical operations. However, we saw responses in which students performed the necessary operations but calculated the incorrect eigenvalues due to simple algebraic errors. These students were coded as correct for the first criterion and incorrect for the second. The third factor is observed for the investigation of the students' interpretation skill. We identified whether students explicitly acknowledged the frequency symbolically and related it to the eigenvalue. The numerical values of the frequencies were not taken in consideration since they do not reflect literacy in interpreting symbols.

\section{RESULTS AND DISCUSSION}

A summary of our analysis of the student responses is shown in Table II. The table presents our assessment of the three skills in the student responses; the questions are categorized by their respective skills along with the criteria we used to analyze the responses. Despite extensive instruction on these topics, the tasks proved to be difficult for students, even Tasks 2 and 3 which were posed on the final exam after students had previously seen Task 1 on a midterm exam. The results from the table presents three general claims. 
TABLE II. Results for the analysis of the written responses. N represents the number of students who appropriately applied the skill.

\begin{tabular}{|c|c|c|c|}
\hline Skill & Analysis & \multicolumn{2}{|c|}{ Results } \\
\hline \multirow{3}{*}{ Mathematization } & \multirow{2}{*}{ General Matrix Structure } & T1 A $\left(\mathrm{N}_{\text {TOTAL }}=53\right)$ & T3 $\left(\mathbf{N}_{\text {TOTAL }}=38\right)$ \\
\hline & & $52 \% \quad(\mathrm{~N}=28)$ & $34 \% \quad(\mathrm{~N}=13)$ \\
\hline & Correct Eigenequation & $49 \% \quad(\mathrm{~N}=26)$ & $29 \% \quad(\mathrm{~N}=11)$ \\
\hline \multirow[b]{2}{*}{ Interpretation } & \multirow[b]{2}{*}{ Symbolic Interpretation of Frequency } & T1 B $\left(\mathbf{N}_{\text {TOTAL }}=53\right)$ & T2 $\left(\mathbf{N}_{\text {TOTAL }}=38\right)$ \\
\hline & & $52 \% \quad(\mathrm{~N}=28)$ & $92 \% \quad(\mathrm{~N}=35)$ \\
\hline \multirow{3}{*}{$\begin{array}{l}\text { Technical } \\
\text { Operation }\end{array}$} & \multirow{2}{*}{ Correct Eigenvalues } & T1 B $\left(\mathbf{N}_{\text {TOTAL }}=53\right)$ & T2 $\left(\mathbf{N}_{\text {TOTAL }}=38\right)$ \\
\hline & & $49 \% \quad(\mathrm{~N}=26)$ & $63 \% \quad(\mathrm{~N}=24)$ \\
\hline & $\begin{array}{l}\text { Execution of Operations } \\
\text { - }(A-\lambda I) \\
\text { - } \operatorname{det}(A-\lambda I)=0\end{array}$ & $73 \% \quad(\mathrm{~N}=39)$ & $78 \% \quad(\mathrm{~N}=30)$ \\
\hline
\end{tabular}

1. Mathematization was difficult for students, particularly in an unfamiliar context. The percentages for mathematization were the lowest out of the three. In particular, the success rates from Task 1 A to Task 3 dropped $20 \%$, despite Task 3 appearing on the course final. This gives some indication of the challenge of mathematization in physical systems that students are not familiar with. Although the questions are mathematically isomorphic (both were normal modes and had similar procedures in building the matrix equation), the students struggled more in building the matrix equation for the coupled LC circuit. Prior to these exams, the students have practiced with similar questions as to Task 1 , which dealt with coupled springs, but not Task 3, the coupled LC circuit. Consequently, only examining the students' application of mathematization for Task 1 may overestimate the students' ability. The previous exposures to the springs system could have potentially made Task 1 more approachable. The complexity of mathematization becomes more evident by the written responses for Task 3 as very few students correctly mathematized to build the correct matrix equation.

2. Interpreting the result of the calculation should not be taken for granted. As noted, a majority of the students were able to set up the problem correctly with using the characteristic equation and appropriately apply its properties. However, having determined an eigenvalue, only half of the students correctly related this eigenvalue to the frequency, with most incorrectly interpreting frequency as either $m$ or $k$. In several cases, students identified $\lambda$ as the frequency with no relation to $\omega$. It is worth noting that students had much greater success $(92 \%)$ determining the frequency in Task 2 on the final exam than in Task 1B (52\%) which was given on a midterm exam. This may reflect prior exposure to a similar task but is still promising.

3. Technical operation was not the most challenging part of the problems, though some students struggled with symbols in matrix operations. There is a perception that executing the mathematics is a major hurdle for students in upperdivision physics. Our data suggests that is not the case in these tasks. In both Tasks 1B and 2, over $70 \%$ of the students correctly executed the matrix operations. Most of these students also calculated the correct eigenvalues. The most common obstacle in the responses with the incorrect eigenvalues was performing computations with symbols. As an example, one student in this category used the characteristic equation correctly. After taking the determinant, the student struggled with proceeding onto the next steps, writing "I'm not too sure where to go from here. Too many variables than I'm used to in an eigenvalue matrix problem." The addition of symbols hindered some students further from executing the technical operation skill and obtaining the correct eigenvalues and frequencies.

\section{CONCLUSION}

This is our preliminary effort to study student application of matrix algebra and eigentheory in physics. Coding the questions based on the skills presented in the PhysicalMathematical Model led to recognizing what skills needed more attention. Though a majority of the students were proficient with technical computations, our data suggest that mathematizing and interpreting were challenging. This may suggest that instructors should focus more on these strucutral skills as well as the transition to matrix operations with symbols instead of numbers. Our next steps in this project are to design and perform interviews to gain a deeper understanding and authentic view of student reasoning with matrix algebra and eigentheory in various physics contexts.

\section{ACKNOWLEDGMENTS}

The authors wish to express their gratitude and appreciation to the CSUF PER team for their support and encouragement to this project. This work was funded by the NSF grant Nos. PHY1406035 and PHY1912660; the opinions, findings, and views in this paper do not reflect the views of the NSF. 
[1] C. Hillebrand-Viljoen and S. Wheaton, How students apply linear algebra to quantum mechanics, in Physics Education Research Conference 2018, PER Conference (Washington, DC, 2018).

[2] M. S. Sabella and E. F. Redish, Student understanding of topics in linear algebra (2002).

[3] M. Wawro, K. Watson, and W. Christensen, Metarepresentational competence with linear algebra in quantum mechanics, in CERME 10 (Dublin, Ireland, 2017).

[4] C. Larson and M. Zandieh, Three interpretations of the matrix equation ax=b, For the Learning of Mathematics 33, 11 (2013).

[5] C. Andrews-Larson, Conceptualizing matrix multiplication: A framework for student thinking, an historical analysis, and a modeling perspective, ProQuest LLC (2010).

[6] D. Carlson, C. R. Johnson, D. C. Lay, and A. D. Porter, The linear algebra curriculum study group recommendations for the first course in linear algebra, The College Mathematics Journal
24, 41â46 (1993).

[7] F. Henderson, C. Rasmussen, M. Zandieh, M. Wawro, and G. Sweeney, Symbol sense in linear algebra: A start toward eigen theory (2010).

[8] M. Thomas and S. Stewart, Eigenvalues and eigenvectors: Embodied, symbolic and formal thinking, Mathematics Education Research Journal 23, 275 (2011).

[9] E. Redish and E. Kuo, Language of physics, language of math: Disciplinary culture and dynamic epistemology, Science Education 24 (2014).

[10] M. Caballero, B. Wilcox, R. Pepper, and S. Pollock, Acer: A framework on the use of mathematics in upper-division physics (2012).

[11] O. Uhden, R. Karam, M. Pietrocola, and G. Pospiech, Modelling mathematical reasoning in physics education, Science Education 21 (2011). 